\title{
Normalized coprime representations for time-varying linear systems
}

\author{
Markus Mueller and Michael Cantoni
}

\begin{abstract}
By considering the behaviour of stabilizable and detectable, linear time-varying state-space models over doublyinfinite continuous time, we establish the existence of so-called normalized coprime representations for the system graphs; that is, stable and stably left (resp. right) invertible, image (resp. kernel) representations that are normalized with respect to the inner product on $L^{2}(-\infty, \infty)$; this is consistent with the notion of normalization used in the time-invariant setting. The approach is constructive, involving the solution of timevarying differential Riccati equations with single-point boundary conditions at either $+\infty$ or $-\infty$. The contribution lies in accommodating state-space models that may not define an exponential dichotomy.
\end{abstract}

Index Terms-Time-varying systems, normalized coprime factorization, gap metric robustness analysis

\section{INTRODUCTION}

Normalized coprime representations of the graph play an important role in robustness analysis with the gap metric [8], [16]. The corresponding issue of existence of normalized coprime factorizations for time-varying linear state-space models has been considered in several papers over the last two decades [15], [13], [1]. In [15], systems which are uniformly completely controllable and observable, and which define an exponential dichotomy, are considered. In [13], only solutions over $[0, \infty)$ are considered, but more concerningly, the main results lead to the erroneous conclusion that directed gaps are equal for finite-dimensional statespace models [4]. Finally, although not explicitly assumed, the proofs in [1] rely on the state-space model defining an exponential dichotomy, as is necessary for the "plant operator', employed therein, to be well-defined.

In this paper, the existence of normalized, stable and stably invertible (i.e. coprime), image and kernel representations of the graph is established for the class of stabilizable and detectable linear time-varying state-space models. This is achieved without requiring that the models define an exponential dichotomy, thereby extending existing results. The absence of an exponential dichotomy arises, for example, in the case of imaginary axis 'poles' in the special case of timeinvariance. The approach is constructive and involves the solution of time-varying differential Riccati equations with single-point boundary conditions. As may be expected, existence of the required Riccati solutions is established via an optimal control problem. This serves to construct stabilizing

Supported in part by the Australian Research Council (Discovery Projects DP0664225 and DP0880494).

M. Mueller is with the School of Engineering, Computing and Mathematics, University of Exeter, England. Email: m.mueller@ex.ac.uk

M. Cantoni is with the Department of Electrical and Electronic Engineering, The University of Melbourne, Australia. Email: cantoni@unimelb.edu.au state feedback or output injection for the system, in a way that permits characterisation of the graph with objects that define exponentially stable dichotomies. Importantly, such objects can be manipulated in an algebraic fashion to then obtain the main results.

The paper evolves along the following line. First, some preliminaries on linear time-varying state-space models are provided. Then the existence of the required solutions to Riccati equations over doubly-infinite time is considered via optimal control problems. This is then exploited to construct the normalized coprime representations described above.

\section{Preliminaries}

Here we recall several basic notions and results needed throughout the paper, and refer to [6] and [9] for more details.

Define, for $n, m \in \mathbb{N}, \mathcal{C B}^{n, m}$ the set of continuous and bounded matrix valued functions, where the norm of a matrix is induced by the euclidean norm, i.e. for $A \in \mathbb{R}^{n \times m}$ applies $\|A\|=\sup _{x \in \mathbb{R}^{m}}\|A x\| /\|x\|$.

Consider the time-varying linear system

$$
\left.\begin{array}{l}
\dot{x}(t)=A(t) x(t)+B(t) u(t) \\
y(t)=C(t) x(t)+D(t) u(t),
\end{array}\right\}
$$

where, for $n, m, p \in \mathbb{N}$, the matrix valued functions $A \in$ $\mathcal{C B}^{n, n}, B \in \mathcal{C B}^{n, m}, C \in \mathcal{C B}\left(\mathbb{R} \rightarrow \mathbb{R}^{p, n}\right)$ and $D \in \mathcal{C B}^{p, m}$. We will refer to the system by writing the tuple $(A, B, C, D)$. To simplify notation in the following we define the set

$$
\mathcal{M}_{n, m, p}:=\left\{(A, B, C, D) \mid \begin{array}{l|l}
A \in \mathcal{C B}^{n, n}, B \in \mathcal{C B}^{n, m} \\
C \in \mathcal{C B}^{p, n}, D \in \mathcal{C B}^{p, m}
\end{array}\right\}
$$

where $n, m, p \in \mathbb{N}$.

For $t, s \in \mathbb{R}$, we let $\Phi_{A}(t, s)$ denote the transition matrix associated with the homogeneous part of system (1). Recall, that $\Phi_{A}(t, s)$ is defined via

$$
\frac{\mathrm{d}}{\mathrm{d} t} \Phi_{A}(t, s)=A(t) \Phi_{A}(t, s), \quad \Phi_{A}(t, t)=I_{n},
$$

and satisfies $\Phi_{A}(t, \tau) \Phi_{A}(\tau, s)=\Phi_{A}(t, s)$ for all $t, s, \tau \in$ $\mathbb{R}$. Also, recall that for bounded $A$ the homogeneous timevarying system $\dot{x}(t)=A(t) x(t)$ does not have solutions with finite escape time.

One may consider solutions of the initial value problem

$$
\dot{x}(t)=A(t) x(t)+B(t) u(t), \quad x\left(t_{0}\right)=x^{0} \in \mathbb{R}^{n} .
$$

If a solution $x\left(\cdot ; t_{0}, x^{0}, u\right)$ of this problem exists on an interval $\left[t_{0}, t_{1}\right] \subset \mathbb{R}$ for a given input $u \in\left[t_{0}, t_{1}\right] \rightarrow \mathbb{R}^{m}$ and initial condition $x\left(t_{0}\right)=x^{0}$, satisfies

$$
x\left(t ; t_{0}, x^{0}, u\right)=\Phi_{A}\left(t, t_{0}\right) x^{0}+\int_{t_{0}}^{t} \Phi_{A}(t, s) B(s) u(s) \mathrm{d} s,
$$


which also defines the output $y\left(\cdot ; t_{0}, x^{0}, u\right)=$ $C(\cdot) x\left(\cdot ; t_{0}, x^{0}, u\right)$.

Since we will consider systems $(A, B, C, D)$ as operator on $L^{2}$-signal spaces we employ another concept for a solution of a linear time-varying system. First recall the definition for exponentially dichotomic, exponentially stable and exponentially anti-stable evolutions [9, Def. 2.1.]:

Definition 2.1: The matrix valued function $A \in \mathcal{C B}^{n, n}$ defines an exponentially dichotomy if, and only if,

$$
\begin{aligned}
& \exists t_{0} \in \mathbb{R} \exists \varrho \geq 1 \exists \sigma>0 \exists P \in \mathbb{R}^{n \times n} \text { with } P^{2}=P: \\
& \begin{cases}\left\|\Phi_{A}\left(t, t_{0}\right) P \Phi_{A}\left(t_{0}, s\right)\right\| \leq \varrho e^{-\sigma(t-s)}, & t \geq s, \\
\left\|\Phi_{A}\left(t, t_{0}\right)\left(I_{n}-P\right) \Phi_{A}\left(t_{0}, s\right)\right\| \leq \varrho e^{-\sigma(s-t)}, & s \geq t .\end{cases}
\end{aligned}
$$

If $P=I_{n}$ then $A$ defines an exponentially stable evolution. On the other hand, $A$ defines an exponentially anti-stable evolution when $P=0$.

Note that if $A$ defines an exponentially stable or an exponentially anti-stable evolution, resp., then there exist $\varrho \geq 1$ and $\sigma>0$ in (2) independent of $t_{0} \in \mathbb{R}$, and therefore (2) holds for all $t_{0} \in \mathbb{R}$ in these cases.

The system $(A, B, C, D)$ is said to be stabilizable if, and only if, there exists a matrix valued function $F \in \mathcal{C B}^{m, n}$ such that $A+B F$ defines an exponentially stable evolution. $(A, B, C, D)$ is said to be detectable if, and only if, there exists $L \in \mathcal{C B}^{n, p}$ such that $A+L C$ defines an exponentially stable evolution.

We will associate a matrix valued function $F \in \mathcal{C B}^{n, m}$ with the multiplication operator $\mathbf{F}: L^{2}\left(\mathbb{R} \rightarrow \mathbb{R}^{m}\right) \rightarrow$ $L^{2}\left(\mathbb{R} \rightarrow \mathbb{R}^{n}\right),(t \mapsto x(t)) \mapsto(t \mapsto(\mathbf{F} x)(t):=F(t) x(t))$, where, for $n \in \mathbb{N}$,

$$
L^{2}\left(\mathbb{R} \rightarrow \mathbb{R}^{n}\right):=\left\{\begin{array}{c|c}
{\left[\begin{array}{c}
x_{1} \\
\vdots \\
x_{n}
\end{array}\right]} & \begin{array}{l}
x_{i}: \mathbb{R} \rightarrow \mathbb{R} \text { is } \\
\text { Lebesgue measurable and } \\
\int_{-\infty}^{\infty}\left|x_{i}(t)\right|^{2} \mathrm{~d} t<\infty, \\
i \in\{1, \ldots, n\}
\end{array}
\end{array}\right\} .
$$

Note that all operators associated with matrix valued functions are denoted with bold letters to prevent confusion. The adjoint of the multiplication operator $\mathbf{F}$ is $\mathbf{F}^{*}: L^{2}(\mathbb{R} \rightarrow$ $\left.\mathbb{R}^{n}\right) \rightarrow L^{2}\left(\mathbb{R} \rightarrow \mathbb{R}^{m}\right),(t \mapsto z(t)) \mapsto\left(t \mapsto\left(\mathbf{F}^{*} z\right)(t):=\right.$ $\left.F(t)^{\top} z(t)\right)$. The differential operator $\mathcal{D}: \operatorname{dom} \mathcal{D} \rightarrow L^{2}(\mathbb{R} \rightarrow$ $\mathbb{R})$ is defined by

$$
(t \mapsto x(t)) \mapsto\left(t \mapsto(\mathcal{D} x)(t):=\frac{\mathrm{d}}{\mathrm{d} t} x(t)\right),
$$

$\operatorname{dom} \mathcal{D}=\left\{\begin{array}{l|l}x \in L^{2}(\mathbb{R} \rightarrow \mathbb{R}) & \begin{array}{l}x \text { locally absolutely cnts, } \\ \dot{x} \in L^{2}(\mathbb{R} \rightarrow \mathbb{R})\end{array}\end{array}\right\}$ We also consider application of the differential operator to vector valued function, i.e. set $\mathcal{D} x=\left[\mathcal{D} x_{1} \cdots \mathcal{D} x_{n}\right]^{\top}=\dot{x}$ for $x \in L^{2}\left(\mathbb{R} \rightarrow \mathbb{R}^{n}\right)$,

For $A \in \mathcal{C B}^{n, n}$ and the associated multiplication operator, [2, Thm. 1.1.] yields that the operator $\mathcal{D}-\mathbf{A}$ has a bounded inverse on $\operatorname{dom} \mathcal{D}$ if, and only if, $A$ defines an exponentially dichotomic evolution. Moreover, [2, Thm. 1.1.] states that the inverse $(\mathcal{D}-\mathbf{A})^{-1}: L^{2}\left(\mathbb{R} \rightarrow \mathbb{R}^{n}\right) \rightarrow L^{2}\left(\mathbb{R} \rightarrow \mathbb{R}^{n}\right)$ is then given by

$$
(t \mapsto z(t)) \mapsto\left(t \mapsto\left((\mathcal{D}-\mathbf{A})^{-1} z\right)(t)=\int_{-\infty}^{\infty} \widetilde{k}(t, s) z(s) \mathrm{d} s\right),
$$

where

$$
\widetilde{k}(t, s):= \begin{cases}\Phi_{A}\left(t, t_{0}\right) P \Phi_{A}\left(t_{0}, s\right), & t \geq s, \\ -\Phi_{A}\left(t, t_{0}\right)(I-P) \Phi_{A}\left(t_{0}, s\right), & s \geq t,\end{cases}
$$

and $t_{0} \in \mathbb{R}, P \in \mathbb{R}^{n \times n}$ are such that (2) is satisfied.

When $A$ defines an exponentially dichotomic evolution for the linear time-varying state-space model $(A, B, C, D)$ of the form (1), the plant operator $\mathcal{H}=\mathbf{D}+\mathbf{C}(\mathcal{D}-$ $\mathbf{A})^{-1} \mathbf{B}: L^{2}\left(\mathbb{R} \rightarrow \mathbb{R}^{m}\right) \rightarrow L^{2}\left(\mathbb{R} \rightarrow \mathbb{R}^{p}\right)$ is defined by

$$
\begin{aligned}
&(t \mapsto u(t)) \mapsto(t \mapsto y(t)=(\mathcal{H} u)(t) \\
&\left.\quad=\int_{-\infty}^{\infty} k(t, s) u(s) \mathrm{d} s+D(t) u(t)\right)
\end{aligned}
$$

where

$k(t, s):= \begin{cases}C(t) \Phi_{A}\left(t, t_{0}\right) P \Phi_{A}\left(t_{0}, s\right) B(s), & t \geq s, \\ -C(t) \Phi_{A}\left(t, t_{0}\right)(I-P) \Phi_{A}\left(t_{0}, s\right) B(s), & s \geq t,\end{cases}$

and $t_{0} \in \mathbb{R}, P \in \mathbb{R}^{n \times n}$ are such that (2) is satisfied for $A$. The manipulation and composition of systems in this form can be achieved in an algebraic fashion.

Recall that the differential operator $\mathcal{D}$, although unbounded, is densely defined in $L^{2}\left(\mathbb{R} \rightarrow \mathbb{R}^{n}\right)$. Therefore, see $\left[14\right.$, Ch. 13], $\mathcal{D}$ has a linear adjoint $\mathcal{D}^{*}$; see also $[9$, Sec. 2.1.] for more details. Specifically, $\operatorname{dom} \mathcal{D}^{*}=\operatorname{dom} \mathcal{D}$ and $\mathcal{D}^{*} x=-\dot{x}=-\mathcal{D} x$. When $A$ defines an exponentially dichotomic evolution, the solution $x \in L^{2}\left(\mathbb{R} \rightarrow \mathbb{R}^{n}\right)$ of $\dot{x}=A x+f$, for $f \in L^{2}\left(\mathbb{R} \rightarrow \mathbb{R}^{n}\right)$, is in $\operatorname{dom} \mathcal{D}^{*}$ and we have $(\mathcal{D}-\mathbf{A})^{*}=-\mathcal{D}-\mathbf{A}^{*}$ and $(\mathcal{D}-\mathbf{A})^{-*}=\left(-\mathcal{D}-\mathbf{A}^{*}\right)^{-1}$.

In the following we will utilize the concept of duality for linear time-varying linear systems, see [9, Sec. 2] and [11, Sec. 1.8] for example; note that the expression for the dual in [9, Eq. (2.18)] is not entirely consistent with the definitions provided for the operators used to define it; we simply use the definition from [11, Sec. 1.8]. To this end, the following notation is used: for any $B \in \mathcal{C B}^{n, m}$ the matrix valued function $B^{\#}: \mathbb{R} \rightarrow \mathbb{R}^{m \times n}$ is defined such that $B^{\#}(t):=B(-t)^{\top}$ for all $t \in \mathbb{R}$.

\section{OPTIMAL CONTROL AND THE TIME-VARYING DIFFERENTIAL RICCATI EQUATION}

First we introduce the deterministic linear optimal regulator problem for time-varying linear systems, see [11, Sec. 3.3] or [10, Sec. 3]. This is central to our construction of normalized coprime representations of the system graph. The optimal regulator/stabilizer for $A$ is obtained by considering the case of $D=0$. This suffices to construct the representations for models with $D \neq 0$ as shown later.

Definition 3.1: Consider, for $n, m, p \in \mathbb{N}$ and $(A, B, C, 0) \in \mathcal{M}_{n, m, p}$ the linear time-varying system (1) with $D=0$. Then, for $t_{0}, t_{1} \in \mathbb{R}$ with $t_{0}<t_{1}, P_{1} \in \mathbb{R}^{n \times n}$ positive semi-definite, $R_{2} \in \mathcal{C B}^{m, m}$ and $R_{3} \in \mathcal{C B}^{p, p}$ symmetric and positive definite for all $t \in \mathbb{R}$, and the solution $x(\cdot):=x\left(\cdot ; t_{0}, x^{0}, u\right):\left[t_{0}, t_{1}\right] \rightarrow \mathbb{R}^{n}$ and output $y(\cdot):=y\left(\cdot ; t_{0}, x^{0}, u\right):\left[t_{0}, t_{1}\right] \rightarrow \mathbb{R}^{p}$ of (1) with initial 
condition $x\left(t_{0}\right)=x^{0}$, the deterministic linear optimal regulator problem can be expressed as:

$$
\begin{aligned}
\inf _{u \in \mathcal{C}^{1}\left(\left[t_{0}, t_{1}\right] \rightarrow \mathbb{R}^{m}\right)}\left\{\int _ { t _ { 0 } } ^ { t _ { 1 } } \left[y(s)^{\top} R_{3}(s) y(s)\right.\right. \\
\left.\left.+u(s)^{\top} R_{2}(s) u(s) \mathrm{d} s\right]+x\left(t_{1}\right)^{\top} P_{1} x\left(t_{1}\right)\right\} .
\end{aligned}
$$

We denote the input which achieves the optimum of (3) by $t \mapsto u^{\text {opt }}(t)$ and for $t_{1} \in \mathbb{R}, P_{1} \in \mathbb{R}^{n \times n}, \tau \in\left[t_{0}, t_{1}\right]$ and $x^{\tau}:=x\left(\tau ; t_{0}, x^{0}, u^{\text {opt }}\right) \in \mathbb{R}^{n}$, define

$$
\begin{aligned}
\left(\tau, x^{\tau}, t_{1}, P_{1}\right) \mapsto V^{\mathrm{opt}}\left(\tau, x^{\tau}, t_{1}, P_{1}\right) \\
:=\int_{\tau}^{t_{1}}\left[y(s)^{\top} R_{3}(s) y(s)+\left(u^{\mathrm{opt}}(s)\right)^{\top} R_{2}(s) u^{\mathrm{opt}}(s) \mathrm{d} s\right] \\
\quad+x\left(t_{1}\right)^{\top} P_{1} x\left(t_{1}\right),
\end{aligned}
$$

where $x(\cdot)$ and $y(\cdot)$ are the solution of (1) for the optimal input $u^{\mathrm{opt}}$ and initial condition $x\left(t_{0}\right)=x^{0} \in \mathbb{R}^{n}$.

A solution for the deterministic linear optimal regulator problem (3) is obtained by solving a certain time-varying Riccati equation.

Proposition 3.2: Let $t_{1} \in \mathbb{R}$. For the time-varying linear system $(A, B, C, 0) \in \mathcal{M}_{n, m, p}$, of the form (1), there exist a $t_{0}<t_{1}$ such that the optimal control $u^{\text {opt }}:\left[t_{0}, t_{1}\right] \rightarrow \mathbb{R}^{m}$ for (3) is given by

$$
u^{\mathrm{opt}}(t)=-R_{2}(t)^{-1} B(t)^{\top} P\left(t ; t_{1}, P_{1}\right) x(t),
$$

where $P\left(\cdot ; t_{1}, P_{1}\right):\left[t_{0}, t_{1}\right] \rightarrow \mathbb{R}^{n \times n}$ is the solution of the single-point boundary-value problem

$$
\begin{aligned}
-\frac{\mathrm{d}}{\mathrm{d} t} P(t)= & R_{1}(t)+A(t)^{\top} P(t)+P(t) A(t) \\
& -P(t) B(t) R_{2}(t)^{-1} B(t)^{\top} P(t), P\left(t_{1}\right)=P_{1},
\end{aligned}
$$

and $R_{1}(t):=C(t)^{\top} R_{3}(t) C(t)$. Moreover, for all $\tau \in$ $\left[t_{0}, t_{1}\right]$, any initial condition $x\left(t_{0}\right)=x^{0} \in \mathbb{R}^{n}$ for (1), and $x^{\tau}=x\left(\tau ; t_{0}, x^{0}, u^{\mathrm{opt}}\right) \in \mathbb{R}^{n}$,

$$
V^{\mathrm{opt}}\left(\tau, x^{\tau}, t_{1}, P_{1}\right)=\left(x^{\tau}\right)^{\top} P\left(\tau ; t_{1}, P_{1}\right) x^{\tau} .
$$

A proof is omitted here. We refer the reader to [12] which holds detailed proofs for all statements of the present note.

Proposition 3.3: For all $t_{1} \in \mathbb{R}$ and all symmetric and positive semi-definite $P_{1} \in \mathbb{R}^{n \times n}$, the Riccati initial value problem (5) has a unique solution

$$
P:\left(-\infty, t_{1}\right] \rightarrow \mathbb{R}^{n \times n}, \quad t \mapsto P\left(t ; t_{1}, P_{1}\right) .
$$

Proposition 3.4: If the system $(A, B, C, D)$ is stabilizable then there exists a bounded solution

$$
\Pi: \mathbb{R} \rightarrow \mathbb{R}^{n \times n}, t \mapsto \Pi(t)=\Pi(t ; \infty, 0):=\lim _{t_{1} \rightarrow \infty} P\left(t ; t_{1}, 0\right)
$$

of the Riccati equation (5) with boundary condition $\lim _{t_{1} \rightarrow \infty} P\left(t_{1}\right)=0$.

See [12] for proofs for Props. 3.3 and 3.4.

It remains to show that applying the control law $u^{\text {opt }}(t)=$ $-R_{2}(t)^{-1} B(t)^{\top} \Pi(t ; \infty, 0) x(t)$ to (1) yields a stable system, i.e. that $A(\cdot)-B(\cdot) R_{2}(\cdot)^{-1} B(\cdot)^{\top} \Pi(\cdot ; \infty, 0)$ defines an exponentially stable evolution.
Proposition 3.5: If the time-varying linear system $(A, B, C, D)$ is stabilizable and detectable, and if $R_{2} \in \mathcal{C B}^{m, m}$ and $R_{3} \in \mathcal{C B}^{p, p}$ are positive definite for all $t \in \mathbb{R}$, then

$$
t \mapsto A(t)-B(t) R_{2}(t)^{-1} B(t)^{\top} \Pi(t ; \infty, 0)
$$

defines an exponentially stable evolution; $\Pi(\cdot ; \infty, 0)$ here is the solution of the Riccati equation (5) with single-point boundary condition $\lim _{t_{1} \rightarrow \infty} P\left(t_{1}\right)=0$.

To prove this we use the following lemma.

Lemma 3.6: If the time-varying linear system $(A, B, C, D)$ is detectable and if, for any $t_{0} \in \mathbb{R}$, $u \in L^{2}\left(\left[t_{0}, \infty\right) \rightarrow \mathbb{R}^{m}\right)$ and $y \in L^{2}\left(\left[t_{0}, \infty\right) \rightarrow \mathbb{R}^{p}\right)$ then the solution $x\left(\cdot ; t_{0}, x^{0}, u\right)$ of (1) with initial condition $x\left(t_{0}\right)=x^{0} \in \mathbb{R}^{n}$ is in $L^{2}\left(\left[t_{0}, \infty\right) \rightarrow \mathbb{R}^{n}\right)$ and there exist $c_{1}, c_{2}>0$ such that

$$
\begin{aligned}
& \left\|x\left(\cdot ; t_{0}, x^{0}, u\right)\right\|_{L^{2}\left[t_{0}, \infty\right)}^{2} \\
& \quad \leq c_{1}\left\|x^{0}\right\|^{2}+c_{2}\left(\|u\|_{L^{2}\left[t_{0}, \infty\right)}^{2}+\|y\|_{L^{2}\left[t_{0}, \infty\right)}^{2}\right) .
\end{aligned}
$$

See [12] for a proof.

Proof of Prop. 3.5. Recall function $V^{\text {opt }}$ from Def. 3.1. In view of Prop. 3.2 we have, for $t_{1}=\infty$ and $P_{1}=0$, that

$$
\begin{aligned}
& \left(x^{0}\right)^{\top} \Pi\left(t_{0} ; \infty, 0\right) x^{0}=V^{\mathrm{opt}}\left(t_{0}, x^{0}, \infty, 0\right) \\
& \quad=\int_{t_{0}}^{\infty} y(s)^{\top} R_{3}(s) y(s)+u^{\mathrm{opt}}(s)^{\top} R_{2}(t) u^{\mathrm{opt}}(s) \mathrm{d} s,
\end{aligned}
$$

where $\Pi(\cdot ; \infty, 0)$ is a solution of the Riccati equation (5) with single-point boundary condition $\lim _{t_{1} \rightarrow \infty} P\left(t_{1}\right)=0$. Boundedness of $\Pi(\cdot ; \infty, 0)$ (see Prop. 3.4) and positive definiteness of $R_{2}(t)$ and $R_{2}(t)$ for all $t \in \mathbb{R}$, gives the existence of $c_{1}>0$ such that

$$
\|y\|_{L^{2}\left[t_{0}, \infty\right)}^{2} \leq c_{1}\left\|x^{0}\right\|^{2} \text { and }\left\|u^{\text {opt }}\right\|_{L^{2}\left[t_{0}, \infty\right)}^{2} \leq c_{1}\left\|x^{0}\right\|^{2} \text {. }
$$

Thus, Lem. 3.6 yields the existence of a constant $c_{2}>0$ such that

$$
\left\|x\left(\cdot ; t_{0}, x^{0}, u^{\mathrm{opt}}\right)\right\|_{L^{2}\left[t_{0}, \infty\right)}^{2} \leq c_{2}\left\|x^{0}\right\|^{2} .
$$

Since, in view of $u^{\text {opt }}=F x$ with $F:=-R_{2}^{-1} B^{\top} \Pi(\cdot ; \infty, 0)$, we have $x\left(\cdot ; t_{0}, x^{0}, u^{\mathrm{opt}}\right)=\Phi_{A+B F}\left(\cdot, t_{0}\right) x^{0}$, and since (9) holds for arbitrary $x^{0} \in \mathbb{R}^{n}$, in particular for all unit vectors, we obtain that the $L^{2}$-norm of all rows of $\Phi_{A+B F}\left(\cdot, t_{0}\right)$ is bounded by $c_{2}$, hence there exists a constant $c_{3}>0$ independent of $t_{0}$ and $t_{1}$ such that

$$
\int_{t_{0}}^{t_{1}}\left\|\Phi_{A+B F}\left(t, t_{0}\right)\right\|^{2} \mathrm{~d} t \leq c_{3}
$$

for all $t_{1} \geq t_{0}$. Now, we may conclude the proof applying [3, Thm. 29.3.]: inequality (10) is sufficient for that $A+B F$ defines an exponential stable evolution.

To summarize, we have the following result.

Corollary 3.7: If the time-varying linear system $(A, B, C, D)$ is stabilizable then the optimal control $u^{\text {opt }}: \mathbb{R} \rightarrow \mathbb{R}^{m}$ for the deterministic linear optimal regulator problem (3) on $\mathbb{R}$ is given by

$$
u^{\mathrm{opt}}(t)=\underbrace{-R_{2}(t)^{-1} B(t)^{\top} \Pi(t ; \infty, 0)}_{=: F(t)} x(t),
$$


where $\Pi(\cdot ; \infty, 0)$ is the solution of the Riccati equation (5) with boundary condition $\lim _{t_{1} \rightarrow \infty} P\left(t_{1}\right)=0$ and $R_{2}$ is given as in Prop. 3.2. Moreover, if $(A, B, C, D)$ is detectable then $A+B F$ defines an exponentially stable evolution. In this case we call the solution $\Pi(\cdot ; \infty, 0)$ of the Riccati singlepoint boundary-value problem stabilizing.

This result is used to construct normalized right coprime representations of the graph of the system $(A, B, C, D)$. Normalized left coprime representations are obtained via a similar result, obtained by considering the so-called dual

$$
\left.\begin{array}{rl}
\dot{z}(t) & =A(-t)^{\top} z(t)+C(-t)^{\top} v(t) \\
w(t) & =B(-t)^{\top} z(t)+D(-t)^{\top} v(t)
\end{array}\right\} ;
$$

see $[11$, Sec. 1.8$]$. This is denoted by $\left(A^{\#}, C^{\#}, B^{\#}, D^{\#}\right)$.

Lemma 3.8: (i) The time-varying bounded matrix $A \in$ $\mathcal{C B}^{n, n}$ defines an exponentially stable evolution if, and only if, $A^{\#}$ defines an exponentially stable evolution. (ii) The time-varying system $(A, B, C, D)$ of form (1) is stabilizable (detectable) if, and only if, the dual system $\left(A^{\#}, C^{\#}, B^{\#}, D^{\#}\right)$ of form (12) is detectable (stabilizable).

See [12] for a proof.

Applying Cor. 3.7 to the dual system (12) - under the assumption that $\left(A^{\#}, C^{\#}, B^{\#}, D^{\#}\right)$ is stabilizable - gives us an optimal control

$$
v^{\mathrm{opt}}(t)=-\widetilde{R}_{2}(-t)^{-1} C(-t) \widetilde{\Psi}(t ; \infty, 0) z(t)
$$

for the corresponding deterministic linear optimal regulator problem, where $t \mapsto \widetilde{\Psi}(t ; \infty, 0)$ is the solution of the Riccati single-point boundary-value problem

$$
\begin{aligned}
& -\frac{\mathrm{d}}{\mathrm{d} t} \widetilde{Q}(t)=\widetilde{R}_{1}(-t)+A(-t) \widetilde{Q}(t)+\widetilde{Q}(t) A(-t)^{\top} \\
& \quad-\widetilde{Q}(t) C(-t)^{\top} \widetilde{R}_{2}(-t)^{-1} C(-t) \widetilde{Q}(t), \lim _{t_{1} \rightarrow \infty} \widetilde{Q}\left(t_{1}\right)=0,
\end{aligned}
$$

and where $\widetilde{R}_{1}(-t):=B(-t) \widetilde{R}_{3}(-t) B(-t)^{\top}, \widetilde{R}_{2} \in \mathcal{C} \mathcal{B}^{p, p}$ and $\widetilde{R}_{3} \in \mathcal{C B}^{m, m}$ are symmetric and positive definite for all $t \in \mathbb{R}$.

We may rewrite the Riccati equation (13) by setting $Q(t)=\widetilde{Q}(-t)$. This implies $\dot{Q}(t)=-\dot{\widetilde{Q}}(-t)$. Applying $\tau=-t$, transforms (13) into

$$
\begin{aligned}
& \frac{\mathrm{d}}{\mathrm{d} \tau} Q(\tau)=\widetilde{R}_{1}(\tau)+A(\tau) Q(\tau)+Q(\tau) A(\tau)^{\top} \\
& \quad-Q(\tau) C(\tau)^{\top} \widetilde{R}_{2}(\tau)^{-1} C(\tau) Q(\tau), \lim _{\tau_{0} \rightarrow-\infty} Q\left(\tau_{0}\right)=0 .
\end{aligned}
$$

This leads to the optimal control

$$
v^{\mathrm{opt}}(t)=\underbrace{-\widetilde{R}_{2}(-t)^{-1} C(-t) \Psi(-t ;-\infty, 0)}_{=: L^{\#}(t)} z(t)
$$

where $\Psi(\cdot ;-\infty, 0)$ is the solution of Riccati equation (14) with single-point boundary condition $\lim _{t_{0} \rightarrow-\infty} Q\left(t_{0}\right)=0$. In view of the second part of Cor. 3.7 we obtain that, if $\left(A^{\#}, C^{\#}, B^{\#}, D^{\#}\right)$ is detectable, $A^{\#}+C^{\#} L^{\#}$ defines an exponentially stable evolution. Thus, in view of Lem. 3.8(i) and for $L(t)=-\Psi(t ;-\infty, 0) C(t)^{\top} \widetilde{R}_{2}(t)^{-1}, A+L C$ also defines an exponentially stable evolution. As such, the solution $\Psi(\cdot ;-\infty, 0)$ of the Riccati single-point boundaryvalue problem (14) is called stabilizing.

\section{MAIN RESULT: NORMALIZED COPRIME REPRESENTATIONS FOR THE SYSTEM GRAPH}

In the previous section, we consider linear time-varying state-space models $(A, B, C, D)$ that are stabilizable and detectable, but do not require these to define exponentially dichotomic evolutions. This remains the case in what follows. As such, the class of systems considered is larger than in the related papers [15], [1], as discussed in the introduction.

Theorem 4.1: For $n, m, p \in \mathbb{N}$, consider the time-varying linear system $(A, B, C, D) \in \mathcal{M}_{n, m, p}$ of the form (1), and suppose $(A, B, C, D)$ is stabilizable and detectable. Let

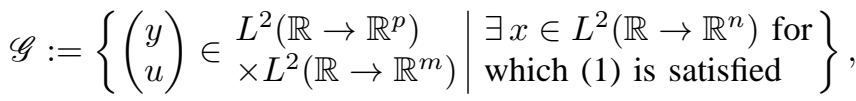

be the system graph, and $R, \widetilde{R} \in \mathcal{C B}^{m, m}$ be defined by $R(t):=I_{m}+D(t)^{\top} D(t)$ and $\widetilde{R}(t):=I_{p}+D(t) D(t)^{\top}$. Let $\mathcal{G}: L^{2}\left(\mathbb{R} \rightarrow \mathbb{R}^{m}\right) \rightarrow L^{2}\left(\mathbb{R} \rightarrow \mathbb{R}^{p} \times \mathbb{R}^{m}\right):=q \mapsto\left(\begin{array}{c}y \\ u\end{array}\right)$ be the operator generated by the stable system

$$
\left.\begin{array}{rl}
\dot{\eta} & =(A+B F) \eta+B R^{-1 / 2} q \\
\left(\begin{array}{c}
y \\
u
\end{array}\right) & =\left(\begin{array}{c}
C+D F \\
F
\end{array}\right) \eta+\left(\begin{array}{c}
D R^{-1 / 2} \\
R^{-1 / 2}
\end{array}\right) q
\end{array}\right\},
$$

where $F:=-R^{-1}\left(B^{\top} \Pi(\cdot ; \infty, 0)+D^{\top} C\right)$ and $\Pi(\cdot ; \infty, 0)$ is the solution of the time-varying Riccati single-point boundary-value problem

$$
\begin{aligned}
-\dot{P}= & {\left[A-B R^{-1} D^{\top} C\right]^{\top} P+P\left[A-B R^{-1} D^{\top} C\right] } \\
& +C^{\top} \widetilde{R}^{-1} C-P B R^{-1} B^{\top} P, \lim _{t_{1} \rightarrow \infty} P\left(t_{1}\right)=0 .
\end{aligned}
$$

Furthermore, let $\widetilde{\mathcal{G}}: L^{2}\left(\mathbb{R} \rightarrow \mathbb{R}^{p} \times \mathbb{R}^{m}\right) \rightarrow L^{2}\left(\mathbb{R} \rightarrow \mathbb{R}^{p}\right):=$ $\left(\begin{array}{c}y \\ u\end{array}\right) \mapsto v$ be the operator generated by the stable system

$$
\left.\begin{array}{l}
\dot{\xi}=(A+L C) \xi+[-L, B+L D]\left(\begin{array}{l}
y \\
u
\end{array}\right) \\
v=\widetilde{R}^{-1 / 2} C \xi+\left[-\widetilde{R}^{-1 / 2}, \widetilde{R}^{-1 / 2} D\right]\left(\begin{array}{l}
y \\
u
\end{array}\right)
\end{array}\right\},
$$

where $L:=-\left(\Psi(\cdot ; \infty, 0) C^{\top}+B D^{\top}\right) \widetilde{R}^{-1}$ and $\Psi(\cdot ;-\infty, 0)$ is the solution of the time-varying Riccati single-point boundary-value problem

$$
\begin{aligned}
\dot{Q}= & {\left[A-B D^{\top} \widetilde{R}^{-1} C\right] Q+Q\left[A-B D^{\top} \widetilde{R}^{-1} C\right]^{\top} } \\
& +B R^{-1} B^{\top}-Q C^{\top} \widetilde{R}^{-1} C Q, \lim _{t_{0} \rightarrow-\infty} Q\left(t_{0}\right)=0 .
\end{aligned}
$$

Then $\mathcal{G}$ is left-invertible by a stable system, $\widetilde{\mathcal{G}}$ is rightinvertible by a stable system and they are both representations of the system graph in the sense that:

$$
\begin{aligned}
\mathscr{G} & =\operatorname{img} \mathcal{G}=\mathcal{G} L^{2}\left(\mathbb{R} \rightarrow \mathbb{R}^{m}\right) ; \\
\mathscr{G} & =\operatorname{ker} \widetilde{\mathcal{G}}=\widetilde{\mathcal{G}}^{-1}(\{0\}) .
\end{aligned}
$$

Moreover, these representations are normalized:

$$
\begin{aligned}
& \mathcal{G}^{*} \mathcal{G}=\mathbf{I} ; \\
& \widetilde{\mathcal{G}} \widetilde{\mathcal{G}}^{*}=\mathbf{I} .
\end{aligned}
$$

Proof: Step 1: In view of (16) and Cor. 3.7 it follows that $F$ is stabilizing for $A-B R^{-1} D^{\top} C$. Similarly, in view of (18), applying Cor. 3.7 to the dual system via Lem. 3.8 and the discussion following this result, we have 
that $L$ is stabilizing for $A-B D^{\top} \widetilde{R}^{-1} C$. Therefore, the systems (15) and (17) that define the operators $\mathcal{G}=:\left[\begin{array}{l}\mathcal{N} \\ \mathcal{M}\end{array}\right]$ and $\widetilde{\mathcal{G}}=:[-\widetilde{\mathcal{M}}, \widetilde{\mathcal{N}}]$ are exponentially stable as claimed.

Step 2.1: We now show that $\mathcal{X}: L^{2}\left(\mathbb{R} \rightarrow \mathbb{R}^{p} \times \mathbb{R}^{m}\right) \rightarrow$ $L^{2}\left(\mathbb{R} \rightarrow \mathbb{R}^{m}\right)$, defined by the system

$$
\begin{aligned}
& \dot{z}=(A+K C) z+[-K, B+K D] w, \\
& p=-R^{1 / 2} F z+\left[0, R^{1 / 2}\right] w,
\end{aligned}
$$

where $K \in \mathcal{C B}^{n, p}$ is such that $A+K C$ defines an exponentially stable evolution, satisfies $\mathcal{X} \mathcal{G}=\mathbf{I}$. Note such $K$ exist since $(A, B, C, D)$ is detectable.

Since $A+B F$ and $A+K C$ define exponentially stable evolutions, the inverses of the operators $\mathcal{D}-(\mathbf{A}+\mathbf{B F})$ and $\mathcal{D}-(\mathbf{A}+\mathbf{K C})$ exist, hence we may write

$$
\begin{aligned}
& \mathcal{G}:=\left[\begin{array}{c}
\mathcal{N} \\
\mathcal{M}
\end{array}\right]:=\left[\begin{array}{c}
\mathbf{D} \mathbf{R}^{-\frac{1}{2}}+(\mathbf{C}+\mathbf{D F})(\mathcal{D}-(\mathbf{A}+\mathbf{B F}))^{-1} \mathbf{B R}^{-\frac{1}{2}} \\
\mathbf{R}^{-\frac{1}{2}}+\mathbf{F}(\mathcal{D}-(\mathbf{A}+\mathbf{B F}))^{-1} \mathbf{B R}^{-\frac{1}{2}}
\end{array}\right], \\
& \mathcal{X}:=\left[0, \mathbf{R}^{\frac{1}{2}}\right]+\mathbf{R}^{\frac{1}{2}} \mathbf{F}(\mathcal{D}-(\mathbf{A}+\mathbf{K C}))^{-1}[\mathbf{K},-(\mathbf{B}+\mathbf{K D})] .
\end{aligned}
$$

Applying the rules of the algebra for operators of systems from $[9$, Sec. 2], we obtain

$$
\begin{aligned}
\mathcal{X G} & =\mathbf{I}+\mathbf{R}^{\frac{1}{2}} \mathbf{F}\left[(\mathcal{D}-(\mathbf{A}+\mathbf{B F}))^{-1}-(\mathcal{D}-(\mathbf{A}+\mathbf{K C}))^{-1}+\right. \\
& \left.(\mathcal{D}-(\mathbf{A}+\mathbf{K C}))^{-1}[\mathbf{K C}-\mathbf{B F}](\mathcal{D}-(\mathbf{A}+\mathbf{B F}))^{-1}\right] \mathbf{B R}^{-\frac{1}{2}} \\
& =\mathbf{I}+\mathbf{R}^{\frac{1}{2}} \mathbf{F}\left[(\mathcal{D}-(\mathbf{A}+\mathbf{B F}))^{-1}-(\mathcal{D}-(\mathbf{A}+\mathbf{K C}))^{-1}+\right. \\
& (\mathcal{D}-(\mathbf{A}+\mathbf{K C}))^{-1}[(\mathcal{D}-(\mathbf{A}+\mathbf{B F}))-(\mathcal{D}-(\mathbf{A}+\mathbf{K C}))] \\
& \left..(\mathcal{D}-(\mathbf{A}+\mathbf{B F}))^{-1}\right] \mathbf{B R}^{-\frac{1}{2}} \\
& =\mathbf{I}
\end{aligned}
$$

Step 2.2: Here we show that $\mathscr{G} \subset \operatorname{img} \mathcal{G}$. Let $\left(\begin{array}{l}y \\ u\end{array}\right) \in \mathscr{G} \subset$ $L^{2}\left(\mathbb{R} \rightarrow \mathbb{R}^{p}\right) \times L^{2}\left(\mathbb{R} \rightarrow \mathbb{R}^{m}\right)$ be arbitrary. Then there exists $x \in L^{2}\left(\mathbb{R} \rightarrow \mathbb{R}^{n}\right)$ such that $\dot{x}=A x+B u$ and $y=C x+D u$. Furthermore, let $q=\mathcal{X}\left(\begin{array}{l}y \\ u\end{array}\right)$. Then there exists $z \in L^{2}(\mathbb{R} \rightarrow$ $\mathbb{R}^{n}$ ) such that

$$
\begin{aligned}
& \dot{z}=(A+K C) z+[-K, B+K D]\left(\begin{array}{l}
y \\
u
\end{array}\right), \\
& q=-R^{1 / 2} F z+\left[0, R^{1 / 2}\right]\left(\begin{array}{c}
y \\
u
\end{array}\right) .
\end{aligned}
$$

In particular, $z=x$, since $A x+B u=(A+K C) x+[-$ $K, B+K D][C x+D u, u]^{\top}$. As such, $q=R^{1 / 2}[u-F x]$ and

$$
\begin{aligned}
\dot{\eta} & =(A+B F) \eta+B R^{-1 / 2} q \\
\left(\begin{array}{c}
y \\
u
\end{array}\right) & =\left(\begin{array}{c}
C+D F \\
F
\end{array}\right) \eta+\left(\begin{array}{c}
D R^{-1 / 2} \\
R^{-1 / 2}
\end{array}\right) q .
\end{aligned}
$$

That is, $\left(\begin{array}{c}y \\ u\end{array}\right) \in \mathcal{G} L^{2}\left(\mathbb{R} \rightarrow \mathbb{R}^{m}\right)$, as claimed.

Step 2.3: We now show the reverse inclusion: $\operatorname{img} \mathcal{G} \subset \mathscr{G}$. Suppose $\left(\begin{array}{l}y \\ u\end{array}\right) \in \operatorname{img} \mathcal{G}$. Then there exists $\eta \in L^{2}\left(\mathbb{R} \rightarrow \mathbb{R}^{n}\right)$ and $q \in L^{2}\left(\mathbb{R} \rightarrow \mathbb{R}^{m}\right)$ such that

$$
\begin{aligned}
\dot{\eta} & =(A+B F) \eta+B R^{-1 / 2} q \\
\left(\begin{array}{c}
y \\
u
\end{array}\right) & =\left(\begin{array}{c}
C+D F \\
F
\end{array}\right) \eta+\left(\begin{array}{c}
D R^{-1 / 2} \\
R^{-1 / 2}
\end{array}\right) q .
\end{aligned}
$$

So we have $u=F \eta+R^{-1 / 2} q$, whereby $y=C \eta+D u$ and $\dot{\eta}=A \eta+B\left(F \eta+R^{-1 / 2} q\right)=A \eta+B u$. Hence, $x=\eta \in$ $L^{2}\left(\mathbb{R} \rightarrow \mathbb{R}^{n}\right)$ satisfies $\dot{x}=A x+B u$ and $y=C x+D u$, whereby $\left(\begin{array}{l}y \\ u\end{array}\right) \in \mathscr{G}$, as required.
Step 3: Here we show that the right coprime representation $\mathcal{G}=\left[{ }_{\mathcal{M}}^{\mathcal{N}}\right]$ is normalized, i.e. $\mathcal{G}^{*} \mathcal{G}=\mathcal{N}^{*} \mathcal{N}+\mathcal{M}^{*} \mathcal{M}=\mathbf{I}$, where $\mathcal{G}^{*}, \mathcal{N}^{*}, \mathcal{M}^{*}$ denote the adjoints with respect to the $L^{2}$ inner-product. Note that:

$\mathcal{N}^{*}=\left(\mathbf{D R}^{-\frac{1}{2}}\right)^{*}+\left(\mathbf{B R}^{-\frac{1}{2}}\right)^{*}\left(-\mathcal{D}-(\mathbf{A}+\mathbf{B F})^{*}\right)^{-1}(\mathbf{C}+\mathbf{D F})^{*} ;$ $\mathcal{M}^{*}=\left(\mathbf{R}^{-\frac{1}{2}}\right)^{*}+\left(\mathbf{B R}^{-\frac{1}{2}}\right)^{*}\left(-\mathcal{D}-(\mathbf{A}+\mathbf{B F})^{*}\right)^{-1} \mathbf{F}^{*}$.

To simplify, we use the short hand $(\mathcal{D}-(\mathbf{A}+\mathbf{B F}))=:(\mathcal{X})$ in what follows. Since $R(t)$ is symmetric for all $t \in \mathbb{R}$, we have $R(t)^{-1 / 2}$ is symmetric for all $t \in \mathbb{R}$. Thus,

$$
\begin{array}{rl}
\mathcal{G}^{*} & \mathcal{G}=\mathcal{N}^{*} \mathcal{N}+\mathcal{M}^{*} \mathcal{M} \\
= & \mathbf{R}^{-\frac{1}{2}} \mathbf{D}^{*} \mathbf{D} \mathbf{R}^{-\frac{1}{2}}+\mathbf{R}^{-\frac{1}{2}} \mathbf{D}^{*}(\mathbf{C}+\mathbf{D F})(\mathcal{X})^{-1} \mathbf{B R}^{-\frac{1}{2}} \\
& +\mathbf{R}^{-\frac{1}{2}} \mathbf{B}^{*}(\mathcal{X})^{-*}\left(\mathbf{C}^{*}+\mathbf{F}^{*} \mathbf{D}^{*}\right) \mathbf{D} \mathbf{R}^{-\frac{1}{2}}+\mathbf{R}^{-\frac{1}{2}} \mathbf{B}^{*}(\mathcal{X})^{-*} \\
& \cdot\left[\mathbf{C}^{*} \mathbf{C}+\mathbf{C}^{*} \mathbf{D F}+\mathbf{F}^{*} \mathbf{D}^{*} \mathbf{C}+\mathbf{F}^{*} \mathbf{D}^{*} \mathbf{D F}\right](\mathcal{X})^{-1} \mathbf{B} \mathbf{R}^{-\frac{1}{2}} \\
& +\mathbf{R}^{-\frac{1}{2}} \mathbf{F}(\mathcal{X})^{-1} \mathbf{B} \mathbf{R}^{-\frac{1}{2}}+\mathbf{R}^{-\frac{1}{2}} \mathbf{B}^{*}(\mathcal{X})^{-*} \mathbf{F}^{*} \mathbf{R}^{-\frac{1}{2}} \\
& +\mathbf{R}^{-\frac{1}{2}} \mathbf{R}^{-\frac{1}{2}}+\mathbf{R}^{-\frac{1}{2}} \mathbf{B}^{*}(\mathcal{X})^{-*} \mathbf{F}^{*} \mathbf{F}(\mathcal{X})^{-1} \mathbf{B} \mathbf{R}^{-\frac{1}{2}}
\end{array}
$$

Now using $\mathbf{R}=\mathbf{I}+\mathbf{D}^{*} \mathbf{D}$ and $\mathbf{F}=-\mathbf{R}^{-\mathbf{1}}\left(\mathbf{B}^{*} \boldsymbol{\Pi}+\mathbf{D}^{*} \mathbf{C}\right)$,

$$
\begin{aligned}
& \mathcal{G}^{*} \mathcal{G} \\
& =\mathbf{R}^{-\frac{1}{2}}[\mathbf{R}+\underbrace{\left[\mathbf{I}-\mathbf{D}^{*} \mathbf{D R}^{-\mathbf{1}}-\mathbf{R}^{-\mathbf{1}}\right]}_{=\mathbf{I}-\mathbf{R} \mathbf{R}^{-1}=0} \mathbf{D}^{*} \mathbf{C}(\mathcal{X})^{-1} \mathbf{B} \\
& +\left[-\mathbf{D}^{*} \mathbf{D R}^{-1}-\mathbf{R}^{-1}\right] \mathbf{B}^{*} \boldsymbol{\Pi}(\mathcal{X})^{-1} \mathbf{B} \\
& +\mathbf{B}^{*}(\mathcal{X})^{-*} \mathbf{C}^{*} \mathbf{D} \underbrace{\left[\mathbf{I}-\mathbf{R}^{-1} \mathbf{D}^{*} \mathbf{D}-\mathbf{R}^{-1}\right]}_{=\mathbf{I}-\mathbf{R}^{-1} \mathbf{R}=0} \\
& +\mathbf{B}^{*}(\mathcal{X})^{-*} \mathbf{\Pi B}\left[-\mathbf{R}^{-\mathbf{1}} \mathbf{D}^{*} \mathbf{D}-\mathbf{R}^{-1} \mathbf{R}=0\right. \\
& +\mathbf{B}^{*}(\mathcal{X})^{-*}\left[\mathbf { C } ^ { * } \left[\mathbf{I}-\mathbf{D R}^{-1} \mathbf{D}^{*}-\mathbf{D} \mathbf{R}^{-1} \mathbf{D}^{*}\right.\right. \\
& +\mathbf{D R}^{-1} \mathbf{D}^{*} \mathbf{D R}^{-1} \mathbf{D}^{*}+\mathbf{D R}^{-1} \mathbf{R}^{-1} \mathbf{D}^{*} \mathbf{C} \\
& +\mathbf{C}^{*} \mathbf{D} \underbrace{\left[-\mathbf{R}^{-1}+\mathbf{R}^{-1} \mathbf{D}^{*} \mathbf{D} \mathbf{R}^{-1}+\mathbf{R}^{-1} \mathbf{R}^{-1}\right]} \mathbf{B}^{*} \boldsymbol{\Pi} \\
& =-\mathbf{R}^{-1}+\mathbf{R}^{-1} \mathbf{R} \mathbf{R}^{-1}=0 \\
& +\Pi B \overbrace{\left[-\mathbf{R}^{-1}+\mathbf{R}^{-1} \mathbf{D}^{*} \mathbf{D R}^{-1}+\mathbf{R}^{-1} \mathbf{R}^{-1}\right]}^{\mathbf{D}^{*} \mathbf{C}} \\
& \left.\left.+\boldsymbol{\Pi B}\left[\mathbf{R}^{-1} \mathbf{D}^{*} \mathbf{D} \mathbf{R}^{-1}+\mathbf{R}^{-1} \mathbf{R}^{-1}\right] \mathbf{B}^{*} \boldsymbol{\Pi}\right](\mathcal{X})^{-1} \mathbf{B}\right] \mathbf{R}^{-\frac{1}{2}} \text {. }
\end{aligned}
$$

Since $R(t)$ is invertible for all $t \in \mathbb{R}$, and omitting the $t$ for convenience,

$$
I_{p}-D R^{-1} D^{\top}=\left[I_{p}+D D^{\top}\right]^{-1}=\widetilde{R}^{-1} .
$$

In view of the Riccati equation (16), this leads to

$$
\begin{aligned}
& \mathcal{G}^{*} \mathcal{G}=\mathbf{R}^{-\frac{1}{2}}[\mathbf{R} \\
& -\mathbf{B}^{*}(\mathcal{X})^{-*}\left(\mathcal{D}-\mathbf{A}+\mathbf{B R}^{-1} \mathbf{B}^{*} \boldsymbol{\Pi}+\mathbf{B R}^{-1} \mathbf{D}^{*} \mathbf{C}\right)^{*} \boldsymbol{\Pi}(\mathcal{X})^{-1} \mathbf{B} \\
& -\mathbf{B}^{*}(\mathcal{X})^{-*} \boldsymbol{\Pi}\left(\mathcal{D}-\mathbf{A}+\mathbf{B R}^{-1} \mathbf{B}^{*} \boldsymbol{\Pi}+\mathbf{B R}^{-1} \mathbf{D}^{*} \mathbf{C}\right)(\mathcal{X})^{-1} \mathbf{B} \\
& +\mathbf{B}^{*}(\mathcal{X})^{-*}\left[\mathbf{C}^{*}\left[\mathbf{I}+\mathbf{D R}^{-1}\left[\mathbf{I}+\mathbf{D}^{*} \mathbf{D}-2 \mathbf{R}\right] \mathbf{R}^{-1} \mathbf{D}^{*}\right] \mathbf{C}\right. \\
& \left.\left.+\mathbf{\Pi B R}^{-1} \mathbf{B}^{*} \boldsymbol{\Pi}\right](\mathcal{X})^{-1} \mathbf{B}\right] \mathbf{R}^{-\frac{1}{2}} \\
& =\mathbf{I}+\mathbf{R}^{-\frac{1}{2}} \mathbf{B}^{*}(\mathcal{X})^{-*}\left[-\mathcal{D}^{*} \boldsymbol{\Pi}-\dot{\mathbf{\Pi}}-\mathbf{\Pi} \mathcal{D}\right](\mathcal{X})^{-1} \mathbf{B R}^{-\frac{1}{2}} .
\end{aligned}
$$

Noting that for every $x \in \operatorname{dom} \mathcal{D}$,

$$
\begin{aligned}
\left(\mathcal{D}^{*} \Pi+\dot{\Pi}+\Pi \mathcal{D}\right)(x) & =-\mathcal{D}(\Pi x)+\dot{\Pi} x+\Pi(\dot{x}) \\
& =-\dot{\Pi} x-\Pi \dot{x}+\dot{\Pi} x+\Pi \dot{x}=0,
\end{aligned}
$$

so that $\mathcal{D}^{*} \boldsymbol{\Pi}+\dot{\boldsymbol{\Pi}}+\boldsymbol{\Pi} \mathcal{D}=\mathbf{0}$, we have that (20a) holds.

Step 4.1: In view of Step 1, $\widetilde{\mathcal{G}}$ defined by the system (17) is stable. We now show that $\mathcal{Y}: L^{2}\left(\mathbb{R} \rightarrow \mathbb{R}^{p}\right) \rightarrow L^{2}(\mathbb{R} \rightarrow$ $\left.\mathbb{R}^{p} \times \mathbb{R}^{m}\right)$, defined by the system

$$
\begin{aligned}
\dot{z} & =(A+B E) z+L \widetilde{R}^{1 / 2} q, \\
\left(\begin{array}{c}
p \\
w
\end{array}\right) & =\left(\begin{array}{c}
C+D E \\
E
\end{array}\right) z+\left(\begin{array}{c}
-\widetilde{R}^{1 / 2} \\
0
\end{array}\right) q,
\end{aligned}
$$


where $E \in \mathcal{C B}^{m, n}$ is such that $A+B E$ defines an exponentially stable evolution, satisfies $\widetilde{\mathcal{G}} \mathcal{Y}=I$. Such $E$ exist since $(A, B, C, D)$ is stabilizable.

Since $A+L C$ and $A+B E$ define exponentially stable evolutions, the inverses of the operators $\mathcal{D}-(\mathbf{A}+\mathbf{L C})$ and $\mathcal{D}-(\mathbf{A}+\mathbf{B E})$ exist, hence we may write

$$
\begin{aligned}
\widetilde{\mathcal{G}} & :=[-\widetilde{\mathcal{M}}, \widetilde{\mathcal{N}}] \\
& :=\widetilde{\mathbf{R}}^{-\frac{1}{2}}[-\mathbf{I}, \mathbf{D}]+\widetilde{\mathbf{R}}^{-\frac{1}{2}} \mathbf{C}(\mathcal{D}-(\mathbf{A}+\mathbf{L C}))^{-1}[-\mathbf{L}, \mathbf{B}+\mathbf{L D}], \\
\mathcal{Y} & :=\left[\begin{array}{c}
-\widetilde{\mathbf{R}}^{\frac{1}{2}}+(\mathbf{C}+\mathbf{D E})(\mathcal{D}-(\mathbf{A}+\mathbf{B E}))^{-1} \mathbf{L} \widetilde{\mathbf{R}}^{\frac{1}{2}} \\
\mathbf{E}(\mathcal{D}-(\mathbf{A}+\mathbf{B E}))^{-1} \mathbf{L} \widetilde{\mathbf{R}}^{\frac{1}{2}}
\end{array}\right] .
\end{aligned}
$$

Following the analysis of Step 2.1,

$$
\begin{aligned}
\widetilde{\mathcal{G}} \mathcal{Y}= & \mathbf{I}+\widetilde{\mathbf{R}}^{-\frac{1}{2}} \mathbf{C}\left[-(\mathcal{D}-(\mathbf{A}+\mathbf{B E}))^{-1}+(\mathcal{D}-(\mathbf{A}+\mathbf{L} \mathbf{C}))^{-1}\right. \\
& \left.+(\mathcal{D}-(\mathbf{A}+\mathbf{L} \mathbf{C}))^{-1}[\mathbf{L} \mathbf{C}-\mathbf{B E}](\mathcal{D}-(\mathbf{A}+\mathbf{B E}))^{-1}\right] \mathbf{B} \widetilde{\mathbf{R}}^{\frac{1}{2}} \\
= & \mathbf{I}
\end{aligned}
$$

Step 4.2: Here we show that $\mathscr{G} \subset \operatorname{ker} \widetilde{\mathcal{G}}$. Suppose $\left(\begin{array}{c}y \\ u\end{array}\right) \in \mathscr{G}$. Then there exists $x \in L^{2}\left(\mathbb{R} \rightarrow \mathbb{R}^{n}\right)$ such that $\dot{x}=A x+B u$ and $y=C x+D u$. So $\xi=x \in L^{2}\left(\mathbb{R} \rightarrow \mathbb{R}^{n}\right)$ satisfies

$$
\begin{aligned}
\dot{\xi} & =A \xi+B u=A \xi+L C \xi-L C x-L D u+B u+L C u \\
& =(A+L C) \xi+[-L, B+L C]\left(\begin{array}{l}
y \\
u
\end{array}\right)
\end{aligned}
$$

and

$$
\begin{aligned}
v & =\widetilde{R}^{-1 / 2} C \xi+\left[-\widetilde{R}^{-1 / 2}, \widetilde{R}^{-1 / 2} D\right]\left(\begin{array}{c}
y \\
u
\end{array}\right) \\
& =\widetilde{R}^{-1 / 2} C \xi-\widetilde{R}^{-1 / 2}[C \xi+D u]+\widetilde{R}^{-1 / 2} D u=0 .
\end{aligned}
$$

Thus $\left(\begin{array}{c}y \\ u\end{array}\right) \in \operatorname{ker} \widetilde{\mathcal{G}}$, as required.

Step 4.3: We now show the reverse inclusion: $\operatorname{ker} \mathcal{G} \subset \mathscr{G}$. Suppose $\left(\begin{array}{c}y \\ u\end{array}\right) \in \operatorname{ker} \widetilde{\mathcal{G}}$. Then there exists $\xi \in L^{2}\left(\mathbb{R} \rightarrow \mathbb{R}^{n}\right)$ such that

$$
\begin{aligned}
& \dot{\xi}=(A+L C) \xi+[-L, B+L D]\left(\begin{array}{l}
y \\
u
\end{array}\right) \\
& 0=\widetilde{R}^{-1 / 2} C \xi-\widetilde{R}^{-1 / 2} y+\widetilde{R}^{-1 / 2} D u .
\end{aligned}
$$

So we have $y=\widetilde{R}^{1 / 2}\left[\widetilde{R}^{-1 / 2} C \xi+\widetilde{R}^{-1 / 2} D u\right]=C \xi+D u$ and $\dot{\xi}=A \xi+L C \xi-L(C \xi+D u)+[B+L D] u=A \xi+B u$, and hence there exists $x=\xi \in L^{2}\left(\mathbb{R} \rightarrow \mathbb{R}^{n}\right)$ which satisfies $\dot{x}=A x+B u$ and $y=C x+D u$. Thus $\left(\begin{array}{l}y \\ u\end{array}\right) \in \mathscr{G}$.

Step 5: Finally we show that the left coprime representation $\widetilde{\mathcal{G}}=[-\widetilde{\mathcal{M}}, \widetilde{\mathcal{N}}]$ is normalized, i.e. $\widetilde{\mathcal{G}} \widetilde{\mathcal{G}}^{*}=\widetilde{\mathcal{M}} \widetilde{\mathcal{M}}^{*}+$ $\widetilde{\mathcal{N}} \widetilde{\mathcal{N}}^{*}=$ I, where $\widetilde{\mathcal{G}}^{*}, \widetilde{\mathcal{N}}^{*}, \widetilde{\mathcal{M}}^{*}$ denote the adjoints with respect to the $L^{2}$ inner-product.

Similar algebraic manipulation (see also [12] for more details) as in Step 3 and application of the Riccati equation (18) leads to

$$
\begin{aligned}
& \widetilde{\mathcal{G}} \widetilde{\mathcal{G}}^{*}=\mathbf{I}+\widetilde{\mathbf{R}}^{-\frac{1}{2}} \mathbf{C}(\mathcal{X})^{-1}[ \\
&-\left(\mathcal{D}-\mathbf{A}+\mathbf{\Psi} \mathbf{C}^{*} \widetilde{\mathbf{R}}^{-1} \mathbf{C}+\mathbf{B D}^{*} \widetilde{\mathbf{R}}^{-1} \mathbf{C}\right) \mathbf{\Psi} \\
&-\mathbf{\Psi}\left(\mathcal{D}-\mathbf{A}+\mathbf{\Psi} \mathbf{C}^{*} \widetilde{\mathbf{R}}^{-1} \mathbf{C}+\mathbf{B D}^{*} \widetilde{\mathbf{R}}^{-1} \mathbf{C}\right)^{*} \\
&\left.+\mathbf{B R} \mathbf{R}^{-1} \mathbf{B}^{*}+\mathbf{\Psi} \mathbf{C}^{*} \widetilde{\mathbf{R}}^{-1} \mathbf{C} \boldsymbol{\Psi}\right](\mathcal{X})^{-*} \mathbf{C}^{*} \widetilde{\mathbf{R}}^{-\frac{1}{2}} \\
&=\mathbf{I}+\widetilde{\mathbf{R}}^{-\frac{1}{2}} \mathbf{C}(\mathcal{X})^{-1}\left[-\mathcal{D} \mathbf{I}+\dot{\Psi}-\mathbf{\Psi} \mathcal{D}^{*}\right](\mathcal{X})^{-*} \mathbf{C}^{*} \widetilde{\mathbf{R}}^{-\frac{1}{2}}
\end{aligned}
$$

For every $x \in \operatorname{dom} \mathcal{D}$, we have

$$
\begin{aligned}
\left(\mathcal{D} \Psi-\dot{\Psi}+\Psi \mathcal{D}^{*}\right)(x) & =\mathcal{D}(\Psi x)-\dot{\Psi} x+\Psi(-\dot{x}) \\
& =\dot{\Psi} x+\Psi \dot{x}-\dot{\Psi} x-\Psi \dot{x}=0
\end{aligned}
$$

and so $\mathcal{D} \boldsymbol{\Psi}-\dot{\boldsymbol{\Psi}}+\boldsymbol{\Psi} \mathcal{D}^{*}=\mathbf{0}$, whence (20b), as claimed. This completes the proof.

\section{CONCLUDing REMARKS}

We have shown that for a time-varying linear system with stabilizable and detectable finite-dimensional state-space realization, there exist normalized coprime representations of the system graph. While the analysis is carried out over doubly-infinite continuous-time, as required to properly define a notion of normalization that is consistent with the time-invariant case, the requirement that the coprime representations be stable, and stably invertible, means they act causally on the singly-infinite time axis, and therefore provide a mechanism for suitably characterising the $L^{2}[0, \infty)$ graph, as well as the $L^{2}(-\infty, \infty)$ graph considered here. This work is part of a broader effort to extend the $\nu$-gap metric robustness analysis framework of [16] to the timevarying setting, with a view to also extending the recent marriage of IQC (integral-quadratic-constraint) and $\nu$-gap metric based analysis in [5] to such a setting.

\section{REFERENCES}

[1] B. D. O. Anderson, T. S. Brinsmead, and F. De Bruyne, "The Vinnicombe metric for nonlinear operators," IEEE Trans. Autom. Control, vol. 47, pp. 1450-1465, 2002.

[2] A. Ben-Artzi and I. C. Gohberg, "Dichotomy of systems and invertibility of linear ordinary differential operators," in Time-variant systems and interpolation, ser. Operator Theory: Advances and Applications, I. C. Gohberg and M. A. Kaashoek, Eds. Birkhäuser, 1992, vol. 56 , pp. $90-119$

[3] R. W. Brockett, Finite Dimensional Linear Systems. New York: John Wiley and Sons Inc., 1970.

[4] M. Cantoni, "On the gap metric for finite-dimensional linear timevarying systems in continuous time," in Control of Uncertain Systems: Modelling, Approximation, and Design, ser. Lecture Notes in Control and Information Sciences, B. A. Francis, M. C. Smith, and J. C. Willems, Eds. Berlin: Springer-Verlag, 2006, vol. 329, pp. 67-78.

[5] M. Cantoni, U. T. Jönsson, and C.-Y. Kao, "IQC robustness analysis for feedback interconnections of unstable distributed parameter systems," in Proc. Joint 48th IEEE Conf. Decis. Control and 28th Chinese Contr. Conf., Shanghai, P.R.China, vol. 1, Shanghai, China, 2009, pp. 1124-1130.

[6] W. A. Coppel, Dichotomies in Stability Theory, ser. Lecture Notes in Mathematics. Berlin, New York: Springer-Verlag, 1978, no. 629.

[7] H. Flanders, "Differentiation under the integral sign," Amer. Math. Monthly, vol. 80, no. 6, pp. 615-627, 1973.

[8] T. T. Georgiou and M. C. Smith, "Optimal robustness in the gap metric," IEEE Trans. Autom. Control, vol. 35, no. 6, pp. 673-686, 1990

[9] V. Ionescu and R. Ştefan, "Generalized time-varying Riccati theory: A Popov operator based approach," Integr. Eq. Oper. Th., vol. 48, pp. 159-212, 2004.

[10] R. E. Kalman, "Contributions on the theory of optimal control," Bol. Soc. Matem. Mexico, vol. II. Ser. 5, pp. 102-119, 1960.

[11] H. Kwakernaak and R. Sivan, Linear Optimal Control Systems. New York: John Wiley and Sons Inc., 1972.

[12] M. Mueller and M. Cantoni, "Normalized coprime representations for linear time-varying systems," submitted, Preprint available: http://empslocal.ex.ac.uk/people/staff/mm411, 2010.

[13] R. Ravi, A. M. Pascoal, and P. P. Khargonekar, "Normalized coprime factorizations for linear time-varying systems," Syst. Control Lett., vol. 18, no. 6, pp. 455-465, 1992.

[14] W. Rudin, Functional Analysis, 2nd ed. New York: McGraw-Hill, 1991

[15] G. Tadmor and M. Verma, "Factorization and the Nehari Theorem in time-varying systems," Math. Control Signals Syst., vol. 5, no. 4, pp. 419-452, 1992.

[16] G. Vinnicombe, Uncertainty and feedback: $\mathcal{H}_{\infty}$ loop-shaping and the $\nu$-gap metric. London: Imperial College Press, 2001.

[17] W. Walter, Ordinary Differential Equations. New York: SpringerVerlag, 1998. 